Original Research Paper

\title{
Optimal Ordering Policy with Stock-Dependent Demand Rate Under Retailer's Two Stages Trade Credit Financing Using Discounted Cash Flow (DCF) Approach
}

\author{
${ }^{1}$ Rakesh Prakash Tripathi, ${ }^{2}$ Harendra Singh and ${ }^{3}$ Neha Sang \\ ${ }^{1}$ Department of Mathematics, Graphic Era University, Dehradun (UK) India \\ ${ }^{2}$ Department of Mathematics, Hindustan College of Science and Technology, Farrah, Mathura (UP) India \\ ${ }^{3}$ Department of Mathematics and Statistics, DDU, Gorakhpur University, (UP) India
}

Article history

Received: 17-06-2015

Revised: 03-10-2015

Accepted: 13-11-2015

Corresponding Author: Rakesh Prakash Tripathi Department of Mathematics, Graphic Era University,

Dehradun (UK) India

Email: tripathi_rp0231@rediffmail.com

\begin{abstract}
Many researchers have assumed one stage trade credit financing. In this study, we considered two levels of trade credit policy using Discounted Cash Flow (DCF) approach. Demand rate is considered to be stock-dependent for the first level (credit demand) and constant for second level (cash demand). Mathematical models are derived under two different circumstances i.e., case I: The permissible delay period is less than or equal to the cycle time and case II: The permissible delay period is greater than or equal to the cycle time for settling the account. An algorithm is provided to determine the optimal order quantity and annual profit. In addition, numerical examples are presented to demonstrate the solution process. Finally, sensitivity analysis of the optimal solution is discussed with respect to different parameters.
\end{abstract}

Keywords: Discounted Cash Flow, Inventory, Two Level Credit Policy, Credit Linked Demand, Credit Period

\section{Introduction}

In classical Economic Order Quantity (EOQ) model, it is assumed that the supplier is paid for the items instantly after they are received. In practice, the supplier permits a certain fixed credit period to settle the account for invigorated retailer's demand. The permissible delay in payment is helpful to attract new customer and increase sales. Inventory models with credit period were first developed by Goyal (1985) to push aside the difference between the selling price and purchase cost. Dave (1985) modified and extended Goyal (1985) model adding the fact that the selling price is necessarily higher than its purchase cost. Haley and Higgins (1973) established the first model to consider the economic order quantity under conditions of permissible delay in payment with deterministic demand. Shah (1993) considered a stochastic inventory model when delays in payments are permissible. Aggarwal and Jaggi (1995) modified Goyal (1985) model for deteriorating items. Jamal et al. (1997) further extended model (1995) allow for shortages. Chang et al. (2003) developed an EOQ model under supplier credits linked to ordering quantity for deteriorating items. Chung and Huang (2003) presented an Economic Production Quantity (EPQ) model for a retailer where the supplier offers a permissible delay in payments. Teng at al. (2012) presented an EOQ model under trade credit financing with increasing demand. Khanra et al. (2011) developed an EOQ model for time dependent demand when delay in payment is permissible. Many researchers like Chu et al. (1998; Chung et al., 2001; Davis and Gaither, 1985; Mandal and Phaujdar, 1989a; Chang et al., 2001; Chung and Liao, 2004; Saiedy and Moghadam, 2011) worked on inventory model by considering delay in payment. Ouyang et al. (2004) presented an inventory model with non instantaneous receipt under permissible delay in payments. Jaggi et al. (2007) developed the retailer's optimal ordering policy under two stage trade credits financing using Discounted Cash Flow (DCF) approach.

In real world, the consumption rate is sometimes affected by the stock level. It is usually observed that a large pile of items on large rack in a supermarket will show the customer to purchase more and then generate demand. The consumption rate may fluctuate with the on hand inventory. Yang et al. (2010) presented an inventory model for deteriorating item with stockdependent demand and partial backlogging. Soni and Shah (2008) established inventory model for retailer when demand is partially constant and partially 
dependent on the stock and the supplier offers progressive credit periods to settle the account. Teng et al. (2011) modified and extended the model (2008) for different situations. Mandal and Phaujdar (1989b) developed a production stock-dependent demand. Two closely related research papers/ articles on stock-dependent rate published by Chang et al. (2010). Alfares (2007) established inventory models in which the demand rate depends on the inventory level and storage time-dependent holding cost. Pal et al. (1991) developed a deterministic inventory model assuming that the demand rate is stock-dependent for deteriorating items. Silver and Peterson (1985) observed that a sale at the retail level is proportional to the amount of inventory displayed. Gupla and Vrat (1986) established inventory model in which demand rate to be a function of initial stock level. Some of the related research in this area are by Wee (1995; Goh, 1994; Ray and Chaudhuri, 1997; Mandal and Maiti, 1999; Dye, 2002; Chung and Tsai, 2001; Yan and Cheng, 1998; Sarker et al., 1997) etc.

At present the effect of inflation plays an important role in any type of business. At present developing countries are facing large scale of inflation due to lock off, strike, natural calamities, political disturbances etc. Thus the effect of inflation cannot be disregarded in real word. Hou (2006) derived an inventory model for deteriorating items with stock-dependent consumption rate and shortages under inflation and time value of money discounting over a finite planning horizon. Ouyang et al. (2002) studied the thump of trade credit in the inventory system. Hou and Lin (2009) developed an inventory model to determine an optimal ordering policy for deteriorating item with delayed payment permitted by the supplier under-inflation and time discounting. Other related research papers/articles were considered by Chang (2004; Chung and Liao, 2006; Jaggi and Aggarwal, 1994; Chapman et al., 1985; Daellenbach, 1986; Haley and Higgins, 1973). Jaggi et al. (2007) determined the retailer's optimal ordering policy under two stage trade credits financing using Discounted Cash Flow (DCF) approach.

Jaggi et al. (2007) developed an inventory model under two levels of trade credit policy by assuming the demand is a function of credit period offered by the retailer to the customer using Discounted Cash Flow (DCF) approach. In this study an attempt is made to formulate the mathematical model for stockdependent credit demand and constant cash demand. The objective function to be maximized is appraised as the retailer's net profit of the inventory system. The effect of parameters on the objective function is discussed numerically. An algorithm is provided to validate the proposed model.

The rest of the paper organized as follows. In the next section, we provide the notations and assumptions for the proposed model. Mathematical formulation is established to manifest retailer's net profit in section 3 . Section 4, provides the optimal solution for finding optimal cycle time. In section 5, algorithm is developed for finding optimal solution. Numerical examples are provided to illustrate the solution algorithm in section 6. In section 7, sensitivity analysis of the optimal solution with respect to different parameters of the system is carried out. Finally, we draw the conclusion and future research in section 8 .

\section{Notations and Assumptions}

The following notations are used through the manuscript:

$\begin{array}{ll}I(t): & \text { The inventory level time 't' } \\ Q: & \text { The order quantity } \\ S: & \text { The ordering cost per order at time zero } \\ c: & \text { The unit purchase cost of the item at time zero } \\ p: & \text { The unit selling price of the item at time zero } \\ i: & \text { Out-of-pocket inventory carrying charge per } \$ \\ & \text { per year } \\ r: & \text { Discount rate per year } \\ I_{e}: & \text { The interest that can be earned per } \$ \text { per year } \\ I_{p}: & \text { The interest charges payable per dollar per } \\ & \left.\text { year ( } \mathrm{I}_{\mathrm{p}}>\mathrm{I}_{\mathrm{e}}\right) \\ m: & \text { Credit period offered to retailer by the } \\ T_{1}: & \text { supplier for settling the accounts } \\ & \text { Credit period granted by the retailer to his/ her } \\ T: & \text { customers; } \mathrm{T}_{1} \leq \mathrm{m} \\ T^{*}: & \text { The inventory cycle time in years } \\ T^{* *}: & \text { Optimal inventory cycle time for case I in years } \\ Z_{1}(T: & \text { Optimal inventory cycle time for case II in years } \\ Z_{2}(T): & \text { Retailer's annual net profit per cycle for case I } \\ Z_{1} *\left(T^{*}\right): & \text { Retailer's annual net profit per cycle for case II } \\ & \text { Optimal retailer's annual net profit per cycle } \\ Z_{2} *\left(T^{* *}\right): & \text { Optimal retailer's annual net profit per cycle } \\ & \text { for case II } \\ Q_{1}: & \text { Order quantity } \\ Q_{1} *: & \text { Optimal order quantity for case I } \\ Q_{2} *: & \text { Optimal order quantity for case II }\end{array}$

\section{Assumptions:}

In addition, the following assumption is being through manuscript:

- Replenishment rate is instantaneous

- Shortages are not allowed

- The annual demand rate consists of (a) regular cash demand and (b) credit demand. Thus demand function at time $\mathrm{t}$ is given by: 


$$
R(t)= \begin{cases}\alpha+\beta I(t) & , 0 \leq \mathrm{t} \leq \mathrm{T}_{1}, 0<\beta \leq 1 \\ \alpha & , \mathrm{T}_{1} \leq \mathrm{t} \leq \mathrm{T}\end{cases}
$$

where, $\alpha$ is known and constant cash-demand rate during the cycle $[0, T]$ and $\beta$ is the credit demand rate during the customer's credit demand rate during the customer's credit period $T_{l}$ :

- The model is considered for one item only

- The Discounted Cash Flow (DCF) approach is applied to consider the various the various cost at various times

- The supplier provides a credit period $m$ to resolve the account to the retailer and retailer passes on a maximum credit $T_{1}$ to its customers to resolve the account. We assume $T_{l} \leq m$ and customer would resolve their account only on last day of the credit period $T_{l}$

\section{Mathematical Formulation}

The inventory is depleted due to demand only. Thus, the rate of change of inventory at time $t$ in $[0, T]$ is given by:

$$
\begin{aligned}
& \frac{d I_{1}(t)}{d t}=-\left\{\alpha+\beta I_{1}(t)\right\}, 0 \leq \mathrm{t} \leq \mathrm{T}_{1} \\
& \frac{d I_{2}(t)}{d t}=-\alpha, \mathrm{T}_{1} \leq \mathrm{t} \leq \mathrm{T}
\end{aligned}
$$

The rate of change of inventory can be easily seen in Fig. 1.

With the boundary condition $\mathrm{I}(0)=\mathrm{Q}, \mathrm{I}(\mathrm{T})=0$, the solution of (1) and (2) is given by:

$$
\begin{aligned}
& I_{1}(t)=\left(Q+\frac{\alpha}{\beta}\right) e^{-\beta t}-\frac{\alpha}{\beta} \\
& I_{2}(t)=\alpha(T-t)
\end{aligned}
$$

and

$$
Q_{1}=\left(Q+\frac{\alpha}{\beta}\right)\left(1-e^{-\beta T_{1}}\right)
$$

$Q_{1}=\alpha\left(T-T_{1}\right)$

Using (5) and (6) in (3), we get:

$$
I_{1}(t)=\alpha\left\{\frac{e^{\beta\left(T_{1}-t\right)}-1}{\beta}+\left(T-T_{1}\right) e^{\beta\left(T_{1}-t\right)}\right\}, 0 \leq \mathrm{t} \leq \mathrm{T}_{1}
$$

and

$$
Q=Q_{1}+Q_{2}=\left(Q+\frac{\alpha}{\beta}\right)\left(1-e^{-\beta T_{1}}\right)+\alpha\left(T-T_{1}\right)
$$

or

$Q=\alpha\left\{\frac{e^{\beta T_{1}}-1}{\beta}+\left(T-T_{1}\right) e^{\beta T_{1}}\right\}$

By using the discounted cash flow approach, the different components of the retailer's net profit is calculated as follows:

The present value of the sales revenue is:

$$
\begin{aligned}
& =\frac{p}{T}\left\{\int_{0}^{T} \alpha e^{-r t} d t+e^{-r T_{1}} \int_{0}^{T_{1}} \beta I_{1}(t) d t\right\} \\
& =\frac{\alpha p}{T}\left[\frac{1-e^{-r T}}{r}+e^{-r T_{1}}\left\{\frac{e^{\beta T_{1}}-1}{\beta}+\left(T-T_{1}\right) e^{\beta T_{1}}-T\right\}\right]
\end{aligned}
$$

The present cost of placing order $=\frac{S}{T}$

The ordering cost $=\frac{c Q}{T}=\frac{c \alpha}{T}\left\{\frac{e^{\beta T_{1}}-1}{\beta}+\left(T-T_{1}\right) e^{\beta T_{1}}\right\}$

The present cost of out of pocket inventory carrying cost is:

$$
\begin{aligned}
& =\frac{i c}{T}\left(\int_{0}^{T_{1}} I_{1}(t) e^{-r T} d t+\int_{T_{1}}^{T} I_{2}(t) e^{-r T} d t\right) \\
& =\frac{i c \alpha}{T}\left[\begin{array}{l}
\left.\left(\frac{1}{\beta}+T-T_{1}\right)\left(\frac{e^{\beta T_{1}}-e^{-r T_{1}}}{\beta+r}\right)+\frac{e^{-r T_{1}}-1}{\beta r}+\frac{1}{r}\right] \\
\left\{\left(T-T_{1}\right) e^{-r T_{1}}+\frac{e^{-r T}-e^{-r T_{1}}}{r}\right\}
\end{array}\right.
\end{aligned}
$$

The following two cases arise which is based on the value of $\mathrm{T}$ and $\mathrm{m}$.

\section{Case $I: m \leq T$}

In this case, the retailer deposits the assembled revenue from cash sales in the period $[0, m]$ and also from credit sales in time period $\left[T_{1}, m\right]$ in to an account that earns interest rate $I_{e}$. At credit period ' $m$ ' credit period, the account have to be resolved, it is assumed that account will be fixed by proceeds of sells produced up to credit period $\mathrm{m}$ and by taking a short term credit at an interest rate of $I_{p}$ in between ( $T$ $m$ ) for financing the remaining stock. Therefore, the present interest earned is: 


$$
\begin{aligned}
& \frac{p I_{e}}{T}\left[\int_{0}^{m} \alpha t e^{-r t} d t+\int_{T_{1}}^{m}\left\{\beta \int I_{1}(t) d t\right\} e^{-r T} d t\right] \\
& =\frac{\alpha p I_{e}}{T}\left[\left\{\frac{1}{r^{2}}\left\{1-(1+r m) e^{-r m}\right\}+\right.\right. \\
& \left.\left\{\frac{e^{\beta T_{1}}-1}{\beta}+\left(T-T_{1}\right) e^{\beta T_{1}}-T\right\}\left(\frac{e^{-r T_{1}}-e^{-r m}}{r}\right)\right]
\end{aligned}
$$

The present interest payable is:

$$
\begin{aligned}
& =\frac{c I_{p}}{T} \int_{m}^{T} I_{2}(t) e^{-r t} d t=\frac{\alpha c I_{p}}{T} \int_{m}^{T}(T-t) e^{-r t} d t \\
& =\frac{\alpha c I_{p}}{r T}\left\{(T-m) e^{-r m}+\frac{e^{-r T}-e^{-r m}}{r}\right\}
\end{aligned}
$$

The retailer's net profit $Z_{l}(T)$ can be expressed as $Z_{l}(T)=$ Sales Revenue + interest earned-purchase costordering Cost-inventory carrying cost-interest payable:

$$
\begin{aligned}
& =\frac{\alpha p}{T}\left[\frac{1-e^{-r T}}{r}+\left\{e^{-r T_{1}}+\frac{I_{e}}{r}\left(e^{-r T_{1}}-e^{-r m}\right)\right\}\left\{\frac{e^{\beta T_{1}}-1}{\beta}+\left(T-T_{1}\right) e^{\beta T_{1}}-T\right\}\right. \\
& \left.+\frac{I_{e}}{r}\left\{\frac{1-(1+r m) e^{-r m}}{r}\right\}\right]-\frac{s}{T}-\frac{c \alpha}{T}\left(\frac{e^{\beta T_{1}}-1}{\beta}+T e^{\beta T_{1}}-T_{1} e^{\beta T_{1}}\right) \\
& -\frac{i c \alpha}{T}\left\{\left(\frac{1}{\beta}+T-T_{1}\right)\left(\frac{e^{\beta T_{1}}-e^{-r T_{1}}}{\beta+r}\right)+\frac{e^{-r T_{1}}-1}{\beta r}+\frac{1}{r}\left(T e^{-r T_{1}}-T_{1} e^{-r T_{1}}+\frac{e^{-r T}-e^{-r T_{1}}}{r}\right)\right\} \\
& -\frac{\alpha c I_{p}}{r T}\left\{(T-m) e^{-r m}+\frac{e^{-r T}-e^{-r m}}{r}\right\}
\end{aligned}
$$

\section{Case II: $m \geq T$}

In this case the credit period $\mathrm{m}$ is longer than or equal to cycle time $T$, therefore the retailer gets interest on each sales during the period $[0, m]$ and also on credit sales in between $\left[T_{l}, m\right]$ and pay no interest for the raw material in stock. The interest earned is:

$$
=\frac{p I_{e}}{T}\left[\int_{0}^{T} \alpha \cdot t \cdot e^{-r T} d t+\int_{T}^{m} I_{2}(0) e^{-r T} d t+\int_{T_{1}}^{m}\left\{\int_{0}^{T} \beta I_{1}(t) d t\right\} e^{-r t} d t\right]=\frac{\alpha p I_{e}}{r T}\left\{\begin{array}{l}
\frac{1-e^{-r T}-r T e^{-r m}}{r}+\left(\frac{e^{\beta T_{1}}-1}{\beta}+T e^{\beta T_{1}}-T_{1} e^{\beta T_{1}}-T\right. \\
\left(e^{-r T_{1}}-e^{-r m}\right)
\end{array}\right\}
$$

Therefore, the retailer's annual net profit $Z_{2}(T)$ is given by $Z_{2}(T)=$ Sales revenue + interest earned-purchase costordering cost-cost of out of pocket inventory carrying cost:

$$
\begin{aligned}
& =\frac{\alpha p}{T}\left[\left(\frac{1-e^{-r T}}{r}\right)\left(1+\frac{I_{e}}{r}\right)+\left\{e^{-r T_{1}}+\frac{I_{e}}{r}\left(e^{-r T_{1}}-e^{-r m}\right)\right\}\left\{\frac{e^{\beta T_{1}}-1}{\beta}+\left(T-T_{1}\right) e^{\beta T_{1}}-T\right\}\right. \\
& \left.-\frac{I_{e} T e^{-r m}}{r}\right]-\frac{s}{T}-\frac{c \alpha}{T}\left\{\frac{e^{\beta T_{1}}-1}{\beta}+\left(T-T_{1}\right) e^{\beta T_{1}}\right\}-\frac{i c \alpha}{T} \\
& {\left[\left(\frac{1}{\beta}+T-T_{1}\right)\left(\frac{e^{\beta T_{1}}-e^{-r T_{1}}}{\beta+r}\right)+\frac{e^{-r T_{1}}-1}{\beta r}+\frac{1}{r}\left\{\left(T-T_{1}\right) e^{-r T_{1}}+\frac{e^{-r T}-e^{-r T_{1}}}{r}\right\}\right]}
\end{aligned}
$$

The present retailer's annual profit, $Z(T)$ can be expressed as:

$$
Z(T)= \begin{cases}Z_{1}(T), & \text { if } \mathrm{m} \leq \mathrm{T} \\ Z_{2}(T), & \text { if } \mathrm{m} \geq \mathrm{T}\end{cases}
$$

At $T=m$,

$Z_{l}(T)=Z_{2}(T)$ 


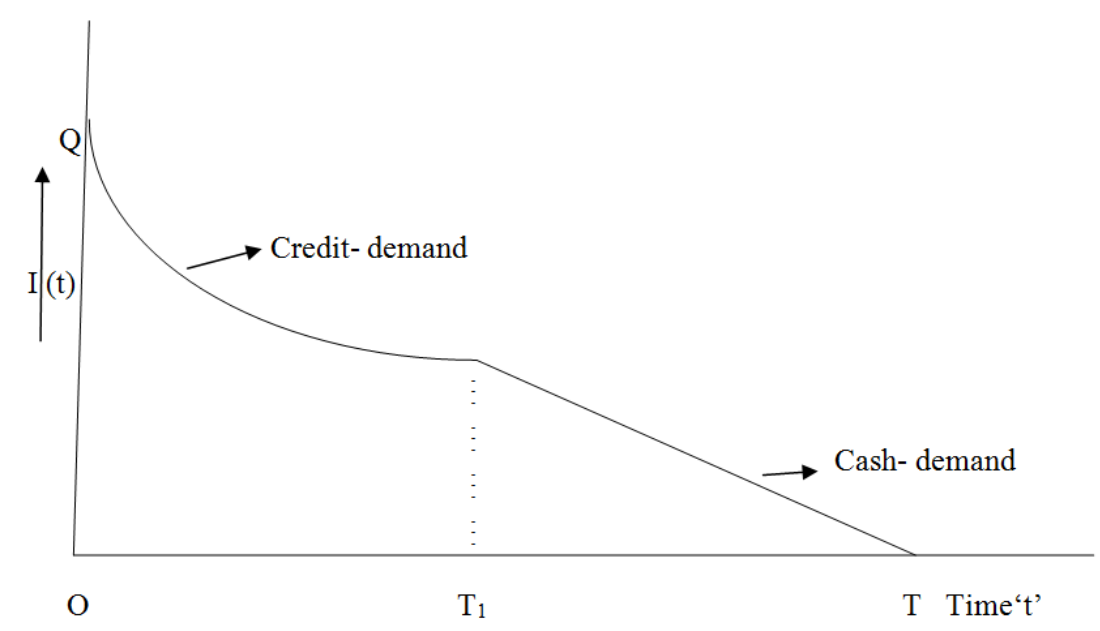

Fig. 1. I(t) Vs time

\section{Determination of Optimal Solution}

To determine optimal value of $T$, taking the first derivative of $Z_{I}(T)$ and $Z_{2}(T)$ with respect to $T$, we obtain:

$$
\begin{aligned}
& \frac{d Z_{1}(T)}{d T}=-\frac{\alpha p}{T^{2}}\left[\frac{1-e^{-r T}}{r}-T e^{-r T}+\left\{e^{-r T_{1}}+\frac{I_{e}}{r}\left(e^{-r T_{1}}-e^{-r m}\right)\right\}\left(\frac{e^{\beta T_{1}}-1}{\beta}-T_{1} e^{\beta T_{1}}\right)\right. \\
& \left.+\frac{I_{e}}{r}\left\{\frac{1-(1+r m) e^{-r m}}{r}\right\}\right]+\frac{s}{T^{2}}+\frac{c \alpha}{T^{2}}\left(\frac{e^{\beta T_{1}}-1}{\beta}-T_{1} e^{\beta T_{1}}\right)+\frac{i c \alpha}{T^{2}}\left\{\left(\frac{1}{\beta}-T_{1}\right)\left(\frac{e^{\beta T_{1}}-e^{-r T_{1}}}{\beta+r}\right)\right. \\
& \left.+\frac{e^{-r T_{1}}-1}{\beta r}+\frac{1}{r}\left(T e^{-r T}-T_{1} e^{-r T_{1}}+\frac{e^{-r T}-e^{-r T_{1}}}{r}\right)\right\}+\frac{\alpha c I_{p}}{r T^{2}}\left\{\left(T+\frac{1}{r}\right) e^{-r T}-\left(m+\frac{1}{r}\right) e^{-r m}\right\}
\end{aligned}
$$

and

$$
\begin{aligned}
& \frac{d Z_{2}(T)}{d T}=-\frac{\alpha p}{T^{2}}\left[\left(\frac{1-e^{-r T}}{r}-T e^{-r T}\right)\left(1+\frac{I_{e}}{r}\right)+\left\{e^{-r T_{1}}+\frac{I_{e}}{r}\left(e^{-r T_{1}}-e^{-r m}\right)\right\}\left(\frac{e^{\beta T_{1}}-1}{\beta}-T_{1} e^{\beta T_{1}}\right)\right]+\frac{s}{T^{2}}+ \\
& \frac{c \alpha}{T^{2}}\left(\frac{e^{\beta T_{1}}-1}{\beta}-T_{1} e^{\beta T_{1}}\right)+\frac{i c \alpha}{T^{2}}\left[\left(\frac{1}{\beta}-T_{1}\right)\left(\frac{e^{\beta T_{1}}-e^{-r T_{1}}}{\beta+r}\right)+\frac{e^{-r T_{1}}-1}{\beta r}+\frac{1}{r}\left\{\left(T+\frac{1}{r}\right) e^{-r T}-\left(T_{1}+\frac{1}{r}\right) e^{-r T_{1}}\right\}\right]
\end{aligned}
$$

Our aim is to find maximum retailer's annual profit. The necessary and sufficient condition to maximize $Z_{i}(T) ; i=1$, 2 , for a given value $T$ are respectively $\frac{d Z_{i}(T)}{d T}=0$ and $\frac{d^{2} Z_{i}(T)}{d T^{2}}>0 ; \mathrm{i}=1,2$. (Appendix).

Now $\frac{d Z_{i}(T)}{d T}=0 ; i=1,2$, give the following equation in $\mathrm{T}$ :

$$
\begin{aligned}
& \alpha p\left[\frac{1-e^{-r T}}{r}-T e^{-r T}+\left\{e^{-r T_{1}}+\frac{I_{e}}{r}\left(e^{-r T_{1}}-e^{-r m}\right)\right\}\left(\frac{e^{\beta T_{1}}-1}{\beta}-T_{1} e^{\beta T_{1}}\right)+\frac{I_{e}}{r}\left\{\frac{1-(1+r m) e^{-r m}}{r}\right\}\right] \\
& -s-c \alpha\left(\frac{e^{\beta T_{1}}-1}{\beta}-T_{1} e^{\beta T_{1}}\right)-i c \alpha\left\{\left(\frac{1}{\beta}-T_{1}\right)\left(\frac{e^{\beta T_{1}}-e^{-r T_{1}}}{\beta+r}\right)+\frac{e^{-r T_{1}}-1}{\beta r}+\frac{1}{r}\left(T e^{-r T}-T_{1} e^{-r T_{1}}+\frac{e^{-r T}-e^{-r T_{1}}}{r}\right)\right\} \\
& -\frac{\alpha c I_{p}}{r}\left\{\left(T+\frac{1}{r}\right) e^{-r T}-\left(m+\frac{1}{r}\right) e^{-r m}\right\}=0
\end{aligned}
$$

and 


$$
\begin{aligned}
& \alpha p\left[\left(\frac{1-e^{-r T}}{r}-T e^{-r T}\right)\left(1+\frac{I_{e}}{r}\right)+\left\{e^{-r T_{1}}+\frac{I_{e}}{r}\left(e^{-r T_{1}}-e^{-r m}\right)\right\}\left(\frac{e^{\beta T_{1}}-1}{\beta}-T_{1} e^{\beta T_{1}}\right)\right] \\
& -s-c \alpha\left(\frac{e^{\beta T_{1}}-1}{\beta}-T_{1} e^{\beta T_{1}}\right)-i c \alpha\left[\left(\frac{1}{\beta}-T_{1}\right)\left(\frac{e^{\beta T_{1}}-e^{-r T_{1}}}{\beta+r}\right)+\frac{e^{-r T_{1}}-1}{\beta r}+\frac{1}{r}\left\{\left(T+\frac{1}{r}\right) e^{-r T}-\left(T_{1}+\frac{1}{r}\right) e^{-r T_{1}}\right\}\right]=0
\end{aligned}
$$

To get the optimal cycle time $T=T^{*}$ for case I and $T=T^{* *}$ for case II, we have to solve Equations (21) and (22), for which $\frac{d^{2} Z_{i}(T)}{d T^{2}}<0$. for $\mathrm{i}=1,2$ (Appendix).

Since it is difficult to solve above Equations (21) and (22), for finding the exact value of $T$, therefore, we make use of the second order approximations for exponential terms, i.e., $e^{-r T}=1-r T+\frac{r^{2} T^{2}}{2}, \quad e^{\beta T_{1}}=1-\beta T_{1}+\frac{\beta^{2} T_{1}^{2}}{2} \quad$ and $e^{-r T_{1}}=1-r T_{1}+\frac{r^{2} T_{1}^{2}}{2}$ etc.

Hence Equations (21) and (22) reduces to:

$$
\begin{aligned}
& \alpha p\left[\frac{r(1-r T) T^{2}}{2}-\frac{\beta T_{1}^{2}}{2}\left\{1-r T_{1}+\frac{r^{2} T_{1}^{2}}{2}+I_{e}\left(T_{1}-m\right)\left(\frac{r T_{1}}{2}+\frac{r m}{2}-1\right)\right\}\left(1+\beta T_{1}\right)+\frac{I_{e} m^{2}}{2}(1-r m)\right] \\
& -s+\frac{c \alpha \beta T_{1}^{2}}{2}\left(1+\beta T_{1}\right)+\frac{i c \alpha}{2}\left\{T_{1}^{2}\left(1+\beta T_{1}-r T_{1}\right)-\left(T-T_{1}\right)\right\}\left\{r\left(T^{2}+T T_{1}+T_{1}^{2}\right)-\left(T+T_{1}\right)\right\} \\
& -\frac{\alpha c I_{p}}{2}\left\{m^{2}(1-r m)-T^{2}(1-r T)\right\}=0
\end{aligned}
$$

and

$$
\begin{aligned}
& \alpha p\left[\frac{r(1-r T) T^{2}}{2}\left(1+\frac{I_{e}}{r}\right)-\frac{\beta T_{1}^{2}}{2}\left\{1-r T_{1}+\frac{r^{2} T_{1}^{2}}{2}+I_{e}\left(T_{1}-m\right)\left(\frac{r T_{1}}{2}+\frac{r m}{2}-1\right)\right\}\left(1+\beta T_{1}\right)\right] \\
& -s+\frac{c \alpha \beta T_{1}^{2}}{2}\left(1+\beta T_{1}\right)+\frac{i c \alpha}{2}\left\{\beta T_{1}^{3}+T^{2}(1-r T)\right\}=0
\end{aligned}
$$

Again, we make use of the second order approximations for exponential terms, i.e., $e^{-r T}=1-r T+\frac{r^{2} T^{2}}{2}$, $e^{\beta T_{1}}=1-\beta T_{1}+\frac{\beta^{2} T_{1}^{2}}{2}$ and $e^{-r T_{1}}=1-r T_{1}+\frac{r^{2} T_{1}^{2}}{2}$ etc.

Hence Equations (8) (15) and (17) reduce to:

$$
\begin{aligned}
& Q=\alpha\left\{T\left(1+\beta T_{1}+\frac{\beta^{2} T_{1}^{2}}{2}\right)-\frac{\beta T_{1}^{2}}{2}\left(1+\beta T_{1}\right)\right\} \\
& Z_{1}(T)=\frac{\alpha p}{T}\left[T\left(1-\frac{r T}{2}\right)+\left\{1-r T_{1}+\frac{r^{2} T_{1}^{2}}{2}+I_{e}\left(T_{1}-m\right)\left(\frac{r T_{1}}{2}+\frac{r m}{2}-1\right)\right\} \frac{\beta T_{1}}{2}\left(2 T-T_{1}+\beta T T_{1}-\beta T_{1}^{2}\right)+\frac{I_{e} m^{2}}{2}(1-r m)\right] \\
& -\frac{s}{T}-\frac{c \alpha}{T}\left\{\left(T-\frac{\beta T_{1}^{2}}{2}\right)\left(1+\beta T_{1}\right)+\frac{\beta^{2} T_{1}^{2} T}{2}\right\}-\frac{i c \alpha}{2 T}\left\{T^{2}+\beta T_{1}^{2}\left(T-T_{1}\right)\right\}-\frac{\alpha c I_{p}(T-m)}{2 T}\{T-m(1-r m)\}
\end{aligned}
$$

and

$$
\begin{aligned}
& Z_{2}(T)=\frac{\alpha p}{T}\left[T\left(1-\frac{r T}{2}\right)\left(1+\frac{I_{e}}{r}\right)+\left\{1-r T_{1}+\frac{r^{2} T_{1}^{2}}{2}+I_{e}\left(T_{1}-m\right)\left(\frac{r T_{1}}{2}+\frac{r m}{2}-1\right)\right\} \frac{\beta T_{1}}{2}\left(2 T-T_{1}+\beta T T_{1}-\beta T_{1}^{2}\right)-\frac{I_{e} T}{r}\left(1-r m+\frac{r^{2} m^{2}}{2}\right)\right] \\
& -\frac{s}{T}-\frac{c \alpha}{T}\left\{\left(T-\frac{\beta T_{1}^{2}}{2}\right)\left(1+\beta T_{1}\right)+\frac{\beta^{2} T_{1}^{2} T}{2}\right\}-\frac{i c \alpha}{2 T}\left\{T^{2}+\beta T_{1}^{2}\left(T-T_{1}\right)\right\}
\end{aligned}
$$


Now, we summarize the above results and establish the following algorithm to find the optimal solution.

\section{Algorithm}

The following steps are to be followed to find optimal annual profit and order quantity:

Step 1: Determine $T^{*}$ from (23), if $T \geq m$, evaluate $Z_{l}\left(T^{*}\right)$, from $(26)$

Step 2: Determine $T^{* *}$ from (24), if $T<m$, evaluate $Z_{2}\left(T^{* *}\right)$, from $(27)$

Step 3: If the condition $T^{*} \geq m$ and $T^{* *}<m$ is satisfied, go to step 4, otherwise go to step 5

Step 4: Compare $Z_{I}\left(T^{*}\right)$ and $Z_{2}\left(T^{*}\right)$ and find the maximum profit

Step 5: If $T^{*}>m$ is satisfied but $T^{* *}>m$, then $Z_{l}\left(T^{*}\right)$ the maximum profit, else if $T^{*}<m$, but $T^{* *}<$ $m$, then $Z_{2}\left(T^{* *}\right)$ is the maximum profit

\section{Numerical Examples}

\section{Example 1: (Case I \& II) Maximum Retailer's Annual Profit $Z_{2}^{*}\left(T^{* *}\right)$}

The following data is considered for inventory system:

- $\alpha=1000$ units per year, $\beta=0.1, r=13 \%, m=5.0$ year, $T_{l}=.5$ year, $c=\$ 20 /$ unit, $i=0.15, I_{e}=9 \%, I_{p}$ $=14 \%, s=\$ 700 /$ unit, $p=\$ 60 /$ unit. Solving Equation (23), we get $T=T^{*}=7.67797$ years, the corresponding values of $Q=Q_{I}^{*}=12087.5$ units and maximum retailer's annual profit $Z_{l}(T)=$ $Z_{1}^{*}\left(T^{*}\right)=\$ 8058.34$

- Again solving Equation (24), we have $T=T^{* *}=$ 0.531856 year, the corresponding values of $Q=Q_{2}$ * $=545.989$ units and maximum retailer's annual $\mathrm{Z}_{2}(\mathrm{~T})=\mathrm{Z}_{2} *\left(\mathrm{~T}^{* *}\right)=\$ 53980.6$

- $\quad$ Here $T^{*}>m$ and $T^{* *}<m$ and $Z_{1}^{*}\left(T^{*}\right)<Z_{2}^{*}\left(T^{* *}\right)$. Hence the maximum average profit in this case is $Z_{2}^{*}\left(T^{* *}\right)=\$ 53980.6$. Where optimal cycle time is $T=T^{*}=0.531856$ year

- The economic order quantity is $Q=Q_{2}{ }^{*}=$ 545.989 units

Example 2: (case I) Maximum Retailer's Annual Profit $Z_{l}^{*}\left(T^{*}\right)$

The following data is considered for inventory system:

- $\alpha=1000$ units per year, $\beta=0.1, r=13 \%, m=$ 0.0822 year, $T_{1}=0.0274$ year, $c=\$ 50 /$ unit, $i=0.15$, $I_{e}=9 \%, I_{p}=14 \%, s=\$ 500 /$ unit, $p=\$ 60 /$ unit. Solving Equation (23), we get $T=T^{*}=0.225374$ year, the corresponding values of $Q=Q_{1}{ }^{*}=$ 225.955 units and maximum retailer's annual profit $Z_{1}(T)=Z_{1}^{*}\left(T^{*}\right)=\$ 5843.36$
- Again solving Equation (24), we have $T=T^{* *}=$ 0.431152 , the corresponding values of $Q=Q_{2}{ }^{*}=$ 432.297 units and maximum retailer's annual $Z_{2}(T)$ $=Z_{2} *\left(T^{* *}\right)=\$ 4837.67$

- Here $T^{* *}>m$ which contradicts case II, only case I holds as $T^{*}>m$. Hence the maximum average profit in this case is $Z_{1} *\left(T^{*}\right)=\$ 5843.36$. Where optimal cycle time is $T=T^{*}=0.225374$ year

- The economic order quantity is $Q=Q_{1} *=$ 225.955 units

Example 3: (Case II) Maximum Retailer's Annual Profit $Z_{2}^{*}\left(T^{* *}\right)$

The following data is considered for inventory system:

- $\alpha=1000$ units per year, $\beta=0.1, r=13 \%, m=0.8$ year, $T_{l}=0.4$ year, $c=\$ 50 /$ unit, $i=0.15, I_{e}=9 \%, I_{p}$ $=14 \%, s=\$ 500 /$ unit, $p=\$ 60 /$ unit. Solving Equation (23), we get $T=T^{*}=0.371669$ year, the corresponding values of $Q=Q_{1} *=378.513$ units and maximum retailer's annual profit $Z_{l}(T)=$ $Z_{1}^{*}\left(T^{*}\right)=\$ 8439.39$

- Again solving Equation (24), we have $T=T^{* *}=$ 0.437502 , the corresponding values of $Q=Q_{2}{ }^{*}=$ 447.032 units and maximum retailer's annual profit $Z_{2}(T)=Z_{2}^{*}\left(T^{* *}\right)=\$ 8624.52$

- Here $\mathrm{T}^{*}<\mathrm{m}$ which contradicts case I, only case II holds as $T^{* *}<m$. Hence the maximum retailer's annual profit in this case is $Z_{2}^{*}\left(T^{* *}\right)=\$ 8624.52$, where optimal cycle time is $T=T^{* *}=0.437502$ year

- The economic order quantity is given by $Q=Q_{2}{ }^{*}=$ 447.032 units

\section{Sensitivity Analysis}

By using the same data as in example 1, we study the effect of the changes in a single parameter keeping other parameters same on the optimal solution as shown in following Tables 1-8.

The following inferences can be made from the results obtained from Tables 1-8:

- When the cash demand ' $\alpha$ ' increases, the order quantity $\left(Q_{l}\right)$ and net profit $Z_{l}(T)$ will also increase. Similarly if the credit demand ' $\beta$ ' increases, the order quantity $\left(Q_{l}\right)$ slightly increases and net profit $Z_{l}(T)$ increases. That is, change in ' $\alpha$ ' will lead the positive change in $Q_{l}$ and $Z_{l}(T)$. The change in ' $\beta$ ' will lead slight change in $Q_{l}$ and change in $Z_{l}(T)$

- When the cash demand ' $\alpha$ ' increases, order quantity $\left(Q_{I}\right)$ and net profit $Z_{I}(T)$ will also increase. Similarly in purchase cost ' $c$ ' increases, the order quantity $\left(Q_{I}\right)$ and net profit $Z_{l}(T)$ will also increase. That is, change in ' $c$ ' will lead the positive change in $\left(Q_{l}\right)$ and $Z_{l}(T)$ 
- When the cash demand ' $\alpha$ ' increases, order quantity $\left(Q_{I}\right)$ and net profit $Z_{I}(T)$ will also increase. Similarly if selling price ' $p$ ' increases, order quantity $\left(Q_{l}\right)$ decreases while net profit $Z_{l}(T)$ increases. That is, change in ' $\alpha$ ' leads positive change in $\left(Q_{l}\right)$ and $Z_{l}(T)$ and the change in ' $\beta$ ' causes negative change in $\left(Q_{l}\right)$ and positive change in $Z_{l}(T)$
- When the cash demand ' $\alpha$ ' increases, order quantity $\left(Q_{l}\right)$ and net profit $Z_{l}(T)$ will also increase. Similarly if ordering $\operatorname{cost}^{\prime} s$ ' increases, order quantity $\left(Q_{l}\right)$ increases while net profit $Z_{l}(T)$ decreases. That is, change in ' $\alpha$ ' leads positive change in $\left(Q_{l}\right)$ and negative change in $Z_{l}(T)$ and the change in ' $s$ ' causes negative change in both $\left(Q_{l}\right)$ and $Z_{l}(T)$

Table 1. Variation of cash demand ' $\alpha$ ' and credit demand ' $\beta$ '

\begin{tabular}{llrrrrrr}
\hline$\alpha \downarrow$ & $\beta \rightarrow$ & \multicolumn{1}{c}{0.2} & \multicolumn{1}{c}{0.3} & 0.4 & \multicolumn{1}{c}{0.6} & 0.7 \\
\hline 1000 & $T$ & 0.225457 & 0.225541 & 0.225625 & 0.225709 & 0.225794 & 0.22588 \\
& $Q$ & 226.620000 & 227.289000 & 227.960000 & 228.632000 & 229.308000 & 229.98600 \\
& $Z_{I}(T)$ & 5869.550000 & 5895.810000 & 5922.130000 & 5948.520000 & 5974.980000 & 6001.50000 \\
1100 & $T$ & 0.215346 & 0.215433 & 0.215522 & 0.215610 & 0.215699 & 0.215788 \\
& $Q$ & 238.099000 & 238.807000 & 239.520000 & 240.233000 & 240.950000 & 241.660000 \\
& $Z_{I}(T)$ & 6696.760000 & 6725.550000 & 6754.410000 & 6783.340000 & 6812.340000 & 6841.420000 \\
1200 & $T$ & 0.260542 & 0.206633 & 0.206725 & 0.206817 & 0.206910 & 0.207003 \\
& $Q$ & 248.485000 & 249.870000 & 250.622000 & 251.375000 & 252.133000 & 252.779000 \\
& $Z_{I}(T)$ & 7534.010000 & 7565.310000 & 7596.690000 & 7628.150000 & 7660.130000 & 7691.310000 \\
1300 & $T$ & 0.206542 & 0.198881 & 0.198976 & 0.199072 & 0.199169 & 0.199265 \\
& $Q$ & 259.744000 & 260.532000 & 261.322000 & 262.116000 & 262.903000 & 263.712000 \\
& $Z_{I}(T)$ & 8380.040000 & 8413.850000 & 8447.740000 & 8481.710000 & 8515.770000 & 8549.920000 \\
1400 & $T$ & 0.191887 & 0.191985 & 0.192084 & 0.192183 & 0.192283 & 0.192383 \\
& $Q$ & 270.012000 & 270.838000 & 271.669000 & 272.501000 & 273.338000 & 274.177000 \\
& $Z_{I}(T)$ & 9233.860000 & 9270.150000 & 9306.540000 & 9343.020000 & 9379.590000 & 9416.260000 \\
1500 & $T$ & 0.185679 & 0.185799 & 0.185901 & 0.186004 & 0.186107 & 0.186211 \\
& $Q$ & 279.936000 & 280.829000 & 281.697000 & 282.569000 & 283.444000 & 284.323000 \\
& $Z_{I}(T)$ & 10094.600000 & 10133.400000 & 10172.300000 & 10211.200000 & 10250.300000 & 10289.500000 \\
\hline
\end{tabular}

Table 2. Variation of cash demand ' $\alpha$ ' and unit purchase cost ' $c$ '

\begin{tabular}{|c|c|c|c|c|c|c|c|}
\hline$\alpha \downarrow$ & $c \rightarrow$ & 45 & 40 & 35 & 30 & 25 & 20 \\
\hline \multirow[t]{3}{*}{1000} & $T$ & 0.232056 & 0.239482 & 0.247801 & 0.257201 & 0.267939 & 0.280359 \\
\hline & $Q$ & 232.65500 & 240.101000 & 248.443000 & 257.869000 & 268.637000 & 281.091000 \\
\hline & $\widetilde{Z}_{I}(T)$ & 10967.40000 & 16096.400000 & 21230.800000 & 26371.400000 & 31519.000000 & 36674.700000 \\
\hline \multirow[t]{3}{*}{1100} & $T$ & 0.221564 & 0.228573 & 0.236423 & 0.245295 & 0.255428 & 0.267149 \\
\hline & $Q$ & 244.348000 & 252.079000 & 260.737000 & 270.523000 & 281.700000 & 294.629000 \\
\hline & $\widetilde{Z}_{I}(T)$ & 12297.100000 & 17931.300000 & 23571.000000 & 29217.200000 & 34870.700000 & 40532.600000 \\
\hline \multirow[t]{3}{*}{1200} & $T$ & 0.212427 & 0.219070 & 0.226511 & 0.234921 & 0.244527 & 0.255637 \\
\hline & $Q$ & 255.567000 & 263.560000 & 272.514000 & 282.634000 & 294.192000 & 307.561000 \\
\hline & $\tilde{Z}_{I}(T)$ & 13636.600000 & 19775.600000 & 25920.500000 & 32071.900000 & 38230.900000 & 44398.600000 \\
\hline \multirow[t]{3}{*}{1300} & $T$ & 0.204376 & 0.210696 & 0.217775 & 0.225777 & 0.234917 & 0.245488 \\
\hline & $Q$ & 266.369000 & 274.607000 & 283.835000 & 294.266000 & 306.181000 & 319.961000 \\
\hline & $Z_{I}(T)$ & 14984.700000 & 21628.300000 & 28278.000000 & 34934.400000 & 41598.600000 & 48271.900000 \\
\hline \multirow[t]{3}{*}{1400} & $T$ & 0.197213 & 0.203244 & 0.210000 & 0.217637 & 0.226361 & 0.236450 \\
\hline & $Q$ & 276.803000 & 285.270000 & 294.754000 & 305.475000 & 317.722000 & 331.886000 \\
\hline & $\widetilde{Z}_{I}(T)$ & 16340.300000 & 23488.400000 & 30642.700000 & 37803.900000 & 44973.100000 & 52151.500000 \\
\hline \multirow[t]{3}{*}{1500} & $T$ & 0.190786 & 0.196557 & 305.312000 & 0.210330 & 0.218679 & 0.228335 \\
\hline & $Q$ & 286.908000 & 295.588000 & 0.203022 & 316.304000 & 328.862000 & 343.386000 \\
\hline & $Z_{I}(T)$ & 17702.800000 & 25355.200000 & 33013.800000 & 40679.600000 & 48353.500000 & 56036.900000 \\
\hline
\end{tabular}

Table 3. Variation of cash demand ' $\alpha$ ' and unit selling price ' $p$ '

\begin{tabular}{|c|c|c|c|c|c|c|c|}
\hline$\alpha \downarrow$ & $p \rightarrow$ & 65 & 70 & 75 & 80 & 85 & 90 \\
\hline \multirow[t]{3}{*}{1000} & $T$ & 0.221683 & 0.218147 & 0.214756 & 0.211500 & 0.208369 & 0.205356 \\
\hline & $Q$ & 222.254000 & 218.708000 & 215.308000 & 212.043000 & 208.903000 & 205.882000 \\
\hline & $\tilde{Z}_{l}(T)$ & 10794.800000 & 15747.300000 & 20701.000000 & 25655.700000 & 30611.500000 & 35568.200000 \\
\hline 1100 & $T$ & 0.211703 & 0.208297 & 0.205030 & 0.201892 & 0.198875 & 0.195971 \\
\hline
\end{tabular}




\begin{tabular}{|c|c|c|c|c|c|c|c|}
\hline & $Q$ & 233.471000 & 229.714000 & 226.110000 & 222.649000 & 219.321000 & 216.118000 \\
\hline & $Z_{1}(T)$ & 12118.400000 & 17570.000000 & 23022.800000 & 28476.700000 & 33931.700000 & 39387.700000 \\
\hline \multirow[t]{3}{*}{1200} & $T$ & 0.203012 & 0.199717 & 0.196557 & 0.193521 & 0.190602 & 0.187792 \\
\hline & $Q$ & 244.238000 & 240.273000 & 236.470000 & 232.817000 & 229.305000 & 225.924000 \\
\hline & $Z_{1}(T)$ & 13452.300000 & 19403.100000 & 25355.200000 & 31308.400000 & 37262.900000 & 43218.400000 \\
\hline \multirow[t]{3}{*}{1300} & $T$ & 0.195354 & 0.192157 & 0.189089 & 0.186142 & 0.183308 & 0.180580 \\
\hline & $Q$ & 254.608000 & 250.441000 & 246.441000 & 242.600000 & 238.905000 & 235.349000 \\
\hline & $Z_{l}(T)$ & 14795.100000 & 21245.300000 & 27696.900000 & 34149.600000 & 40603.700000 & 47058.900000 \\
\hline \multirow[t]{3}{*}{1400} & $T$ & 0.188541 & 0.185429 & 0.182442 & 0.179574 & 0.176814 & 0.174158 \\
\hline & $Q$ & 264.629000 & 260.260000 & 256.067000 & 252.041000 & 248.166000 & 244.437000 \\
\hline & $\bar{Z}_{1}(T)$ & 16145.90000 & 23095.700000 & 30046.800000 & 36999.800000 & 43953.100000 & 50908.100000 \\
\hline \multirow[t]{3}{*}{1500} & $T$ & 0.182428 & 0.179391 & 0.176477 & 0.173678 & 0.170985 & 0.168392 \\
\hline & $Q$ & 274.336000 & 269.768000 & 265.385000 & 261.175000 & 257.125000 & 253.225000 \\
\hline & $\tilde{Z}_{1}(T)$ & 17503.80000 & 24953.200000 & 32404.100000 & 39856.400000 & 47310.100000 & 54765.100000 \\
\hline
\end{tabular}

Table 4. Variation of cash demand ' $\alpha$ ' and ordering $\operatorname{cost}^{\text {' }} \mathrm{s}$ '

\begin{tabular}{|c|c|c|c|c|c|c|c|}
\hline$\alpha \downarrow$ & $s \rightarrow$ & 550 & 600 & 650 & 700 & 750 & 800 \\
\hline \multirow[t]{3}{*}{1000} & $T$ & 0.236001 & 0.246173 & 0.255946 & 0.265364 & 0.274464 & 0.283279 \\
\hline & $Q$ & 236.611000 & 246.811000 & 256.611000 & 266.054000 & 275.179000 & 284.019000 \\
\hline & $Z_{I}(T)$ & 5614.060000 & 5394.830000 & 5184.460000 & 4981.960000 & 4786.500000 & 4597.390000 \\
\hline \multirow[t]{3}{*}{1100} & $T$ & 0.225374 & 0.235055 & 0.244355 & 0.253318 & 0.261978 & 0.270365 \\
\hline & $Q$ & 248.550000 & 257.519000 & 269.487000 & 279.373000 & 288.925000 & 298.176000 \\
\hline & $Z_{1}(T)$ & 6427.700000 & 6197.910000 & 5977.400000 & 5765.120000 & 5560.220000 & 5361.970000 \\
\hline \multirow[t]{3}{*}{1200} & $T$ & 0.216120 & 0.225374 & 0.234263 & 0.242830 & 0.251107 & 0.259123 \\
\hline & $Q$ & 260.010000 & 271.146000 & 281.842000 & 292.150000 & 302.110000 & 311.756000 \\
\hline & $Z_{1}(T)$ & 7251.910000 & 7012.040000 & 6781.840000 & 6560.240000 & 6346.330000 & 6134.360000 \\
\hline \multirow[t]{3}{*}{1300} & $T$ & 0.207968 & 0.216846 & 0.225374 & 0.233591 & 0.241531 & 0.249221 \\
\hline & $Q$ & 271.051000 & 282.624000 & 293.741000 & 304.453000 & 314.803000 & 324.827000 \\
\hline & $Z_{I}(T)$ & 8085.360000 & 7835.840000 & 7596.370000 & 7365.840000 & 7143.290000 & 6927.970000 \\
\hline \multirow[t]{3}{*}{1400} & $T$ & 0.200716 & 0.209259 & 0.217466 & 0.225374 & 0.233014 & 0.240413 \\
\hline & $Q$ & 281.721000 & 293.714000 & 305.235000 & 316.337000 & 327.062000 & 337.449000 \\
\hline & $\bar{Z}_{I}(T)$ & 8927.00000 & 8668.210000 & 8419.830000 & 8180.710000 & 7949.880000 & 7726.520000 \\
\hline \multirow[t]{3}{*}{1500} & $T$ & 0.19421 & 0.202453 & 0.210372 & 0.218002 & 0.225374 & 0.232512 \\
\hline & $Q$ & 292.05800 & 304.456000 & 316.367000 & 327.844000 & 338.932000 & 349.668000 \\
\hline & $Z_{I}(T)$ & 9775.96000 & 9508.230000 & 9251.270000 & 9003.880000 & 8765.050000 & 8533.960000 \\
\hline
\end{tabular}

Sensitivity Analysis (Case II)

Table 5. Variation of cash demand ' $\alpha$ ' and credit demand ' $\beta$ '. (at $s=15$ )

\begin{tabular}{llrrrrrr}
\hline$\alpha \downarrow$ & $\beta \rightarrow$ & \multicolumn{1}{c}{0.2} & 0.3 & 0.4 & 0.5 & \multicolumn{1}{c}{0.6} & 0.7 \\
\hline 1000 & $T$ & 0.0733754 & 0.0736225 & 0.0738738 & 0.0741293 & 0.0743888 & 0.0746524 \\
& $Q$ & 73.5391000 & 73.7869000 & 74.0389000 & 74.2951000 & 74.5553000 & 74.8196000 \\
& $Z_{2}(T)$ & 9523.0200000 & 9543.9600000 & 9564.9500000 & 9585.9800000 & 9607.0700000 & 9628.2100000 \\
1100 & $T$ & 0.0699907 & 0.0702495 & 0.0705127 & 0.0707801 & 0.0710517 & 0.0713275 \\
& $Q$ & 77.1596000 & 77.4451000 & 77.7354000 & 78.0303000 & 78.3299000 & 78.6341000 \\
& $Z_{2}(T)$ & 10523.9000000 & 10546.5000000 & 10569.2000000 & 10592.0000000 & 10614.8000000 & 10637.7000000 \\
1200 & $T$ & 0.0670415 & 0.0673116 & 0.0675860 & 0.0678648 & 0.0681479 & 0.0684352 \\
& $Q$ & 80.6254000 & 80.9504000 & 81.2806000 & 81.6160000 & 81.9567000 & 82.3024000 \\
& $Z_{2}(T)$ & 11526.7000000 & 11551.0000000 & 11575.4000000 & 11599.9000000 & 11624.4000000 & 11649.0000000 \\
1300 & $T$ & 0.0644423 & 0.0647230 & 0.0650082 & 0.0652979 & 0.0655919 & 0.0658902 \\
& $Q$ & 83.9559000 & 84.3218000 & 84.6936000 & 85.0712000 & 85.4545000 & 85.84330000 \\
& $Z_{1}(T)$ & 12531.2000000 & 12557.2000000 & 12583.2000000 & 12609.3000000 & 12635.5000000 & 12661.7000000 \\
1400 & $T$ & 0.0621289 & 0.0624200 & 0.0627155 & 0.0630156 & 0.0633200 & 0.0636288 \\
& $Q$ & 87.1664000 & 87.5751000 & 87.9899000 & 88.4112000 & 88.8385000 & 89.2720000 \\
& $Z_{2}(T)$ & 13537.3000000 & 13564.8000000 & 13592.4000000 & 13620.2000000 & 13648.0000000 & 13675.8000000 \\
1500 & $T$ & 0.0600530 & 0.0603539 & 0.0606594 & 0.0609694 & 0.0612839 & 0.0616028 \\
& $Q$ & 90.2702000 & 90.7288000 & 91.1823000 & 91.6486000 & 92.1216000 & 92.60130000 \\
& $Z_{2}(T)$ & 14544.7000000 & 14573.8000000 & 14603.0000000 & 14632.2000000 & 14661.6000000 & 14691.1000000 \\
\hline
\end{tabular}


Table 6. Variation of cash demand ' $\alpha$ ' and unit purchase cost ' $c$ ' (at $s=15$ )

\begin{tabular}{|c|c|c|c|c|c|c|c|}
\hline$\alpha \downarrow$ & $c \rightarrow$ & 45 & 40 & 35 & 30 & 25 & 20 \\
\hline \multirow[t]{3}{*}{1000} & $T$ & 0.0692223 & 0.0659650 & 0.0632006 & 0.0608191 & 0.0587420 & 0.0569111 \\
\hline & $Q$ & 69.3746000 & 66.1084000 & 63.3364000 & 60.9483000 & 58.8655000 & 57.0296000 \\
\hline & $\tilde{Z}_{2}(T)$ & 14567.7000000 & 19624.6000000 & 24675.1000000 & 29720.6000000 & 34762.2000000 & 39800.5000000 \\
\hline \multirow[t]{3}{*}{1100} & $T$ & 0.0660548 & 0.0629902 & 0.0603911 & 0.0581534 & 0.0562058 & 0.0544845 \\
\hline & $Q$ & 72.8182000 & 69.4379000 & 66.5711000 & 64.1028000 & 61.9513000 & 60.0560000 \\
\hline & $Z_{2}(T)$ & 16070.0000000 & 21629.9000000 & 27183.1000000 & 32731.1000000 & 38274.9000000 & 43815.5000000 \\
\hline \multirow[t]{3}{*}{1200} & $T$ & 0.0632960 & 0.0604009 & 0.0579471 & 0.0558359 & 0.0536203 & 0.0523774 \\
\hline & $Q$ & 76.0342000 & 72.6348000 & 69.6821000 & 67.1418000 & 64.4757000 & 62.9802000 \\
\hline & $Z_{2}(T)$ & 17574.2000000 & 23636.9000000 & 29692.6000000 & 35743.0000000 & 41790.8000000 & 47831.8000000 \\
\hline \multirow[t]{3}{*}{1300} & $T$ & 0.0608652 & 0.0581210 & 0.0557966 & 0.0537979 & 0.0520578 & 0.0505267 \\
\hline & $O$ & 79.2929000 & 75.7157000 & 72.6857000 & 70.8020000 & 67.8119000 & 65.8160000 \\
\hline & $\widetilde{Z}_{2}(T)$ & 19080.1000000 & 25645.4000000 & 32203.6000000 & 38756.2000000 & 45304.5000000 & 51849.3000000 \\
\hline \multirow[t]{3}{*}{1400} & $T$ & 0.0587027 & 0.0560939 & 0.0538858 & 0.0519882 & 0.0503371 & 0.0488852 \\
\hline & $Q$ & 82.3566000 & 78.6942000 & 75.5944000 & 72.9305000 & 70.6126000 & 68.57440000 \\
\hline & $Z_{2}(T)$ & 20587.4000000 & 27655.2000000 & 34715.8000000 & 41770.6000000 & 48821.0000000 & 55867.7000000 \\
\hline \multirow[t]{3}{*}{1500} & $T$ & 0.0567626 & 0.0542767 & 0.0521738 & 0.0503678 & 0.0487973 & 0.0474171 \\
\hline & $Q$ & 85.3211000 & 81.5820000 & 78.4190000 & 75.7025000 & 73.3403000 & 71.2643000 \\
\hline & $Z_{2}(T)$ & 22096.0000000 & 29666.2000000 & 37229.0000000 & 44786.0000000 & 52338.4000000 & 59887.1000000 \\
\hline
\end{tabular}

Table 7. Variation of cash demand ' $\alpha$ ' and unit selling price ' $p$ ' (at $s=15$ )

\begin{tabular}{|c|c|c|c|c|c|c|c|}
\hline$\alpha \downarrow$ & $p \rightarrow$ & 65 & 70 & 75 & 80 & 85 & 90 \\
\hline \multirow[t]{3}{*}{1000} & $T$ & 0.0673476 & 0.0628486 & 0.0592261 & 0.0562323 & 0.0537072 & 0.0515423 \\
\hline & $Q$ & 67.4947000 & 62.9834000 & 59.3510000 & 56.3489000 & 53.8169000 & 51.6461000 \\
\hline & $\tilde{Z}_{2}(T)$ & 14554.6000000 & 19600.1000000 & 24641.0000000 & 29678.8000000 & 34714.5000000 & 39748.6000000 \\
\hline \multirow[t]{3}{*}{1100} & $T$ & 0.0642605 & 0.0600045 & 0.0565795 & 0.0537505 & 0.0513657 & 0.0493221 \\
\hline & $Q$ & 70.8391000 & 66.1446000 & 62.3668000 & 59.2464000 & 56.6159000 & 54.3618000 \\
\hline & $\tilde{Z}_{2}(T)$ & 16058.2000000 & 21607.5000000 & 27152.7000000 & 32694.3000000 & 38233.6000000 & 43771.3000000 \\
\hline \multirow[t]{3}{*}{1200} & $T$ & 0.0615714 & 0.0575284 & 0.0542767 & 0.0515922 & 0.0493305 & 0.0473934 \\
\hline & $Q$ & 74.0432000 & 69.1783000 & 65.2656000 & 62.0353000 & 59.3139000 & 56.9830000 \\
\hline & $Z_{2}(T)$ & 17563.8000000 & 23617.5000000 & 29666.2000000 & 35711.6000000 & 41754.6000000 & 47795.9000000 \\
\hline \multirow[t]{3}{*}{1300} & $T$ & 0.0592019 & 0.0553478 & 0.0522498 & 0.0496936 & 0.0475411 & 0.0456986 \\
\hline & $Q$ & 77.1247000 & 72.1006000 & 68.0622000 & 64.7300000 & 61.9241000 & 59.5222000 \\
\hline & $\tilde{Z}_{2}(T)$ & 19071.1000000 & 25628.8000000 & 32181.5000000 & 38730.6000000 & 45277.2000000 & 51822.1000000 \\
\hline \multirow[t]{3}{*}{1400} & $T$ & 0.0570936 & 0.0534087 & 0.0504484 & 0.0480071 & 0.0459524 & 0.0441946 \\
\hline & $Q$ & 80.0977000 & 74.9246000 & 70.7688000 & 67.3417000 & 64.4572000 & 61.9895000 \\
\hline & $Z_{2}(T)$ & 20579.9000000 & 27641.7000000 & 34698.200000 & 41751.0000000 & 48801.2000000 & 55849.6000000 \\
\hline \multirow[t]{3}{*}{1500} & $T$ & 0.0552021 & 0.0516700 & 0.048834 & 0.0464965 & 0.0445302 & 0.0428488 \\
\hline & $Q$ & 82.9739000 & 77.6612000 & 73.395500 & 69.8797000 & 66.9221000 & 64.3931000 \\
\hline & $\tilde{Z}_{2}(T)$ & 22090.0000000 & 29655.8000000 & 37216.100000 & 44772.6000000 & 52326.5000000 & 59878.4000000 \\
\hline
\end{tabular}

Table 8. Variation of cash demand ' $\alpha$ ' and ordering $\operatorname{cost}^{\prime} s$ '

\begin{tabular}{|c|c|c|c|c|c|c|c|}
\hline$\alpha \downarrow$ & $s \rightarrow$ & 14 & 13 & 12 & 11 & 10 & 5 \\
\hline \multirow[t]{3}{*}{1000} & $T$ & 0.0706576 & 0.06809410 & 0.0654316 & 0.0626576 & 0.0597567 & 0.0424105 \\
\hline & $Q$ & 70.8138000 & 68.24330000 & 65.5735000 & 62.7919000 & 59.8830000 & 42.4892000 \\
\hline & $Z_{2}(T)$ & 9534.5200000 & 9568.08000000 & 9602.9200000 & 9639.2100000 & 9677.1400000 & 9903.4800000 \\
\hline \multirow[t]{3}{*}{1100} & $T$ & 0.0673782 & 0.06493600 & 0.0623994 & 0.0597567 & 0.0569931 & 0.0404705 \\
\hline & $Q$ & 74.2780000 & 71.58420000 & 68.7863000 & 65.8713000 & 62.8230000 & 44.5983000 \\
\hline & $\tilde{Z}_{2}(T)$ & 10535.20 & 570.30000000 & 606.80 & 665.2000000 & 684.6000000 & 10921.6000000 \\
\hline \multirow[t]{3}{*}{1200} & $T$ & 0.0 & 0.06218340 & 567 & 284 & 0.0545845 & 804 \\
\hline & $Q$ & 77.5 & 74.7 & 71.8 & 68. & 000 & 000 \\
\hline & $\bar{Z}_{2}(T)$ & 11537.80 & 11574.50000000 & 11612.6 & 11652.20 & 11693.7000000 & 11940.8 \\
\hline \multirow[t]{3}{*}{1300} & $T$ & 0.0620001 & 0.05975670 & $0.05^{\circ}$ & 0.0549995 & 0.0524612 & 72910 \\
\hline & $Q$ & 80.77 & 77.84790000 & 74.81 & 71.6466000 & 7000 & 48.5624000 \\
\hline & $\widetilde{Z}_{2}(T)$ & 12542.1000000 & 2580.30000000 & 12619.9000000 & 12661.1000000 & 12704.2000000 & 12961.0000000 \\
\hline \multirow[t]{3}{*}{1400} & $T$ & 0.0597567 & 0.05759963 & 0.0553527 & 0.0530152 & 0.0505710 & 0.0359657 \\
\hline & $Q$ & 83.83 & 80.80340000 & 77.6 & $74.3^{\prime}$ & 70.94 & 000 \\
\hline & $\bar{Z}_{2}(T)$ & 13548.0000000 & 13587.50000000 & 13628.6000000 & 13671.8000000 & 13716.0000000 & 13982.0000000 \\
\hline \multirow[t]{3}{*}{1500} & $T$ & 0.0597567 & 0.05565700 & 0.0534908 & 0.0512341 & 0.0488744 & 0.0347766 \\
\hline & & 83.8362000 & 83.65810000 & 80.3999000 & 77.0055000 & 73.4563000 & 52.2516000 \\
\hline & $Z_{2}(T)$ & 14532.4000000 & 14596.10000000 & 14638.5000000 & 14682.7000000 & 14728.9000000 & 15003.8000000 \\
\hline
\end{tabular}




\section{Conclusion and Future Research}

In this study, the retailer's optimal ordering policy under two stage trade credit financing is developed using Discounted Cash Flow (DCF) approach. An algorithm is established to obtain the optimal solution. The sensitivity analysis of the optimal solution with respect to the parameters is also discussed. The results show some phenomena as follows: (i). A higher value of cash demand ' $\alpha$ ' caused higher value of retailer's annual profit, (ii). A higher value of credit demand ' $\beta$ ' causes higher value of retailer's annual profit, (iii). A higher value of unit purchase cost ' $c$ ' causes lower value of retailer's annual profit, (iv). A higher value of selling price ' $p$ ' causes higher value of net profit. That is, the retailer should increase the net profit per transfer from the increase of cash demand, credit demand, purchase cost and selling price. Second order approximation is used for exponential terms to find exact values of cycle time ' $T$ ', order quantity ' $Q$ ' and retailer's annual profit $Z(T)$.

The proposed model can be further extended in several ways. For example, we may add pricing strategy into consideration. We may also extend the model to allow for constant deterioration rate or a two-parameter weibull distribution. In addition, we can consider the demand as a function of time, price as well as quality. Finally, we could generalize the model to allow for shortages, quantity discount or others.

\section{Acknowledgment}

The authors are thankful to anonymous reviewers for valuable comments and to the Editor-in-chief for handling of the manuscript.

\section{Funding Information}

The authors have no support or funding to report.

\section{Author's Contributions}

Rakesh Prakash Tripathi: Paper formation, Mathematical formulation, discussion of data analysis, contributed to the writing of the manuscript and publication.

Harenrda Singh: Coordination of research work and publication of the manuscript.

Neha Sang: Design the research plan, organization, development and publication of the manuscript.

\section{Ethics}

In this paper second order approximation have been used for exponential terms to find closed form optimal solution. With the help of differential calculus the author's have obtained the retailer's annual net profit per unit time.

\section{Appendix A}

To prove this appendix, we first prove the following lemma.

Lemma 1: If a function $G(T)=\frac{F(T)}{T}$, where $\mathrm{F}(\mathrm{T})$ is a differential function of $\mathrm{T}$ two times, then the maximum value of $G(T)$ exist at $T=T^{*}$ if $\frac{1}{T} \frac{d^{2}(F(T))}{d T^{2}}<0$, at $T=T^{*}$.

Proof: It is given that $G(T)=\frac{F(T)}{T}$. For extremum, the necessary condition is $\frac{d(G(T))}{d T}=0$. But $\frac{d(G(T))}{d T}=-\frac{F(T)}{T^{2}}+\frac{1}{T} \frac{d(F(T))}{d T}=\frac{1}{T^{2}}\left(T \frac{d(F(T))}{d T}-F(T)\right)$ $\frac{d(G(T))}{d T}=0$, gives $T \frac{d(F(T))}{d T}-F(T)=0$. (i)

Let Equation (i) be satisfied for $T=T^{*}$.

Again

$\frac{d^{2}(G(T))}{d T^{2}}=-\frac{2}{T^{3}}\left(T \frac{d(F(T))}{d T}-F(T)\right)+\frac{1}{T} \frac{d^{2}(F(T))}{d T^{2}}$

Or $\frac{d^{2}(G(T))}{d T^{2}}=\frac{1}{T} \frac{d^{2}(F(T))}{d T^{2}}$ from (i)

We know that the sufficient condition for existence of a maximum value of $G(T)$ is $\frac{d^{2}(G(T))}{d T^{2}}<0$. Hence the Lemma 1.

Here $Z_{1}(\mathrm{~T})=\frac{1}{\mathrm{~T}}\left[S R+I E_{1}-P C-O C-I C-I P\right]$. For extremum value at $T=T^{*}$

Where Sales revenue $=S R / T$, interest earned $=I E_{I} / T$, Purchase cost $=P C / T$. Ordering cost $=O C / T$, Inventory carrying cost $=I C / T$, Interest payable $=I P / T$.

At $T=T^{*}$, the necessary condition is $\frac{\mathrm{dZ}_{1}(\mathrm{~T})}{\mathrm{dT}}=0$., which gives Equation (18).

If $T=T^{*}$ be a maximum value of $Z_{l}(T)$, then at $T=T^{*}$, we have

$\frac{\mathrm{d}^{2} Z_{1}(\mathrm{~T})}{\mathrm{dT}^{2}}=\frac{1}{\mathrm{~T}}\left[\begin{array}{c}\frac{\mathrm{d}^{2}(\mathrm{SR})}{\mathrm{dT}^{2}}+\frac{\mathrm{d}^{2}\left(\mathrm{IE}_{1}\right)}{\mathrm{dT}^{2}}-\frac{\mathrm{d}^{2}(\mathrm{PC})}{\mathrm{dT}^{2}} \\ -\frac{\mathrm{d}^{2}(O C)}{\mathrm{dT}^{2}}-\frac{\mathrm{d}^{2}(I C)}{\mathrm{dT}^{2}}-\frac{\mathrm{d}^{2}(I P)}{\mathrm{dT}^{2}}\end{array}\right]$. By Lemma 1.

Now at $T=T^{*}$

$\frac{\mathrm{d}^{2} Z_{1}(\mathrm{~T})}{\mathrm{dT}^{2}}=-\frac{\mathrm{re}^{-\mathrm{TT}}}{\mathrm{T}}\left(r p+i c+c I_{p}\right)<0$.

Hence the proof of Appendix A. 


\section{Appendix B:}

We have $\mathrm{Z}_{2}(\mathrm{~T})=\frac{1}{\mathrm{~T}}\left[S R+I E_{2}-P C-O C-I C\right]$.

Where Sales revenue $=S R / T$, interest earned $=I E_{2} / T$, Purchase cost $=P C / T$. Ordering cost $=O C / T$, Inventory carrying cost $=I C / T$.

For extremum value of $Z_{2}(T)$, the necessary condition is $\frac{\mathrm{dZ}_{2}(\mathrm{~T})}{\mathrm{dT}}=0$, which gives Equation (19). If $\mathrm{T}=\mathrm{T}^{* *}$ be a maximum value of $\mathrm{Z}_{2}(\mathrm{~T})$, then at $T=T^{* *}$ $\frac{\mathrm{d}^{2} \mathrm{Z}_{2}(\mathrm{~T})}{\mathrm{dT}^{2}}=\frac{1}{\mathrm{~T}}\left[\frac{\mathrm{d}^{2}(\mathrm{SR})}{\mathrm{dT}^{2}}+\frac{\mathrm{d}^{2}\left(\mathrm{IE}_{2}\right)}{\mathrm{dT}^{2}}-\frac{\mathrm{d}^{2}(\mathrm{PC})}{\mathrm{dT}^{2}}-\frac{\mathrm{d}^{2}(O C)}{\mathrm{dT}^{2}}-\frac{\mathrm{d}^{2}(I C)}{\mathrm{dT}^{2}}\right]$. by Lemma 1. Now at $T=T^{* *}$ $\frac{\mathrm{d}^{2} \mathrm{Z}_{2}(\mathrm{~T})}{\mathrm{dT}^{2}}=-\frac{\mathrm{re}^{-\mathrm{rT}}}{\mathrm{T}}\left\{r p\left(1+\frac{I_{e}}{r}\right)+i c\right\}<0$.

Hence the proof of Appendix B.

\section{References}

Aggarwal, S.P. and C.K. Jaggi, 1995. Ordering policies of deteriorating items under permissible delay in payments. J. Operational Res. Society, 46: 658-662. DOI: $10.1057 /$ jors. 1995.90

Alfares, H.K., 2007. Inventory model with stock-level dependent demand rate and variable holding cost. Int. J. Production Econom., 108: 259-265. DOI: $10.1016 /$ j.ijpe.2006.12.013

Chang, C.T., 2004. An EOQ model with deteriorating items under inflation when supplier credits linked to order quantity. Int. J. Production Econom., 88: 307-316. DOI: 10.1016/S0925-5273(03)00192-0

Chang, C.T., J.T. Teng and S.K. Goyal, 2010. Optimal replenishment policies for non-instantaneous deteriorating items with stock-dependent demand. Int. J. Production Econom., 123: 62-68. DOI: $10.1016 /$ j.ijpe.2009.06.042

Chang, C.T., L.Y. Ouyang and J.T. Teng, 2003. An EOQ model for deteriorating items under supplier credits linked to ordering quantity. Appl. Math. Modell., 27: 983-996. DOI: 10.1016/S0307-904X(03)00131-8

Chang, H.J., C.H. Hung and C.Y. Dye, 2001. An inventory model for deteriorating items with linear trend demand under the condition of permissible delay in payments. Production Plann. Control, 12: 272-282. DOI:10.1080/095372801300107806

Chapman, C.B., S.C. Ward, D.F. Cooper and M.J. Page, 1985. Credit policy and inventory control. J. Operational Res. Society, 35: 1055-1065. DOI: $10.1057 /$ jors. 1984.212

Chu, P., K.J. Chung and S.P. Lan, 1998. Economic order quantity of deteriorating items under permissible delay in payments. Comp. Operations Res., 25: 817-824.

DOI: $10.1016 / \mathrm{S} 0305-0548(98) 00006-9$
Chung, K.J. and J.J. Liao, 2004. Lot-sizing decisions under trade credit depending on the ordering quantity. Comp. Operations Res., 31: 909-928. DOI: $10.1016 / \mathrm{S} 0305-0548(03) 00043-1$

Chung, K.J. and J.J. Liao, 2006. The optimal ordering policy in a DCF analysis for deteriorating items when trade credit depends on the order quantity. Int. J. Production Econom., 100: 116-130. DOI: 10.1016/j.ijpe.2004.10.011

Chung, K.J. and S.F. Tsai, 2001. Inventory systems for deteriorating items with shortages and a linear trend in demand taking account of time value. Comput. Operations Res., 28: 915-934. DOI: $10.1016 / \mathrm{S} 0305-0548(00) 00016-2$

Chung, K.J. and Y.F. Huang, 2003. The optimal cycle time for EPQ inventory model under permissible delay in payments. Int. J. Production Econom., 54: 307-318. DOI: 10.1016/S0925-5273(02)00465-6

Chung, K.J., S.L. Chang and W.D. Yang, 2001. The optimal cycle time for exponentially deteriorating products under trade credit financing. Eng. Economist, 46: 232-242. DOI: $10.1080 / 00137910108967575$

Daellenbach, H.G., 1986. Inventory control and trade credit. J. Operational Res. Society, 37: 525-528. DOI: $10.2307 / 2582676$

Dave, U., 1985. On "Economic order quantity under conditions of permissible delay in payments" by Goyal. J. Operational Res. Society, 46: 1069-1070. DOI: $10.1057 /$ jors. 1985.186

Davis, R.A. and N. Gaither, 1985. Optimal ordering policies under conditions of extended payment privileges. Manage. Sci., 31: 499-509. DOI: org/10.1287/mnsc.31.4.499

Dye, C.Y., 2002. A deterministic inventory model with stock-dependent demand and partial backlogging under condition of permissible delay in payment. Opsearch, 39: 189-201.

Goh, M., 1994. EOQ models with general demand and holding cost functions. Eur. J. Operational Res., 73: 50-54. DOI: 10.1016/0377-2217(94)90141-4

Goyal, S.K., 1985. Economic order quantity under conditions of permissible delay in payments. J. Operational Res. Society, 36: 335-338. DOI: $10.2307 / 2582421$

Gupla, R. and P. Vrat, 1986. Inventory model for stockdependent consumption rate. Opsearch, 23: 19-24.

Haley, C.W. and R.C. Higgins, 1973. Inventory policy and trade credit financing. Manage. Sci., 20: 464-471. DOI: org/10.1287/mnsc.20.4.464

Hou, K.L., 2006. An inventory model for deteriorating items with stock-dependent consumption rate and shortages under inflation and time discounting. Eur. J. Operational Res., 168: 463-474. DOI: 10.1016/j.ejor.2004.05.011 
Hou, K.L. and L.C. Lin, 2009. A cash flow oriented EOQ model with deteriorating items under permissible delay in payments. J. Appl. Sci., 9: 1791-1794. DOI: 10.3923/jas.2009.1791.1794

Jaggi, C.K. and S.P. Aggarwal, 1994. Credit financing in economic ordering policies of deteriorating items. Int. J. Production Econom., 34: 151-155. DOI: $10.1016 / 0925-5273(94) 90031-0$

Jaggi, C.K., K.K. Aggarwal and S.K. Goel, 2007. Retailer's optimal ordering policy under two stage trade credits financing. Adv. Modell. Optimiza., 9: 67-80.

Jamal, A.M.M., B.R. Sarker and S. Wang, 1997. An ordering policy for deteriorating items with allowable shortage and permissible delay in payment. J. Operational Res. Society, 48: 826-833. DOI: $10.1057 /$ palgrave.jors.2600428

Khanra, S., S.K. Ghosh and K.S. Chaudhuri, 2011. An EOQ model for deteriorating item with time dependent quadratic demand under permissible delay in payment. Appl. Math. Comput., 218: 1-9. DOI: $10.1016 /$ j.amc.2011.04.062

Mandal, B.N. and S. Phaujdar, 1989a. An inventory model for deteriorating items and stock-dependent consumption rate. J. Operational Res. Society, 40: 483-488. DOI: 10.1057/jors. 1989.75

Mandal, B.N. and S. Phaujdar, 1989b. Some EOQ models under conditions of permissible delay in payments. Int. J. Manage. Sci., 5: 99-108.

Mandal, M. and M. Maiti, 1999. Inventory of damageable items with variable replenishment rate, stock-dependent demand and some units in hand. Appl. Math. Modell., 23: 799-807.

DOI: $10.1016 / \mathrm{S} 0307-904 X(99) 00018-9$

Ouyang, L.Y. K.W. Chuang and B.R. Chuang, 2004. An inventory model with non-instantaneous receipt and permissible delay in payments. Inform. Manage. Sci., 15: 1-11.

Ouyang, L.Y., M.S. Chen and K.W. Chuang, 2002. Economic Order quantity model under cash discount and payment delay. Informa. Manag. Sci., 13: 1-10.

Pal, S., A. Goswami and K.S. Chaudhuri, 1993. A deterministic inventory model for deteriorating items with stock-dependent demand rate. Int. J. Production Econom., 32: 291-299.

DOI: $10.1016 / 0925-5273(93) 90043-K$
Ray, J. and K.S. Chaudhuri, 1997. An EOQ model with stock-dependent demand, shortage, inflation and time discounting. Int. J. Production Econom., 53: 171-180. DOI: 10.1016/S0925-5273(97)00112-6

Saiedy, H. and M.B. Moghadam, 2011. Inventory model with composed shortage and permissible delay in payment linked to order quantity. J. Ind. Eng. Int., 7: 1-7.

Sarker, B.R., S. Mukherjee and C.V. Balan, 1997. An order level lot size inventory model with inventory level dependent demand and deterioration. Int. J. Pro. Econ., 48: 227-236.

Shah, N.H., 1993. Probabilistic time-scheduling model for an exponentially decaying inventory when delays in payments are permissible. Int. J. Production Econom., 32: 77-82. DOI: 10.1016/0925-5273(93)90009-A

Silver, E.A. and R. Peterson, 1985. Decision Systems For Inventory Management and Production Planning, 2nd Edn., John Wiley and Sons, Singapore, New York. ISBN-10: 0471867829, PP: 736.

Soni, H. and N.H. Shah, 2008. Optimal ordering policy for stock-dependent demand under progressive payment scheme. Eur. J Operational Res, 184: 91-100. DOI: 10.1016/j.ejor.2006.10.048

Teng, J.T., I.P. Krommyda, K. Skouri and K.R. Lon, 2011. A comprehensive extension of optimal ordering policy for stock-dependent demand under progressive payment Scheme. Eur. J. Operational Res., 215: 97-104. DOI: 10.1016/j.ejor.2011.05.056

Teng, J.T., J. Min and Q. Pan, 2012. Economic order quantity model with trade credit financing for nondecreasing demand. Omega, 40: 328-335. DOI: 10.1016/j.omega.2011.08.001

Wee, H.M., 1995. Joint pricing and replenishment policy for deteriorating inventory with declining Market. Int. J. Production Econom., 40: 163-171. DOI: org/10.1016/0925-5273(95)00053-3

Yan, H. and T. Cheng, 1998. Optimal production stopping and restarting times for an EOQ model with deteriorating items. J. Operational Res. Society, 49: 1288-1295. DOI: 10.1057/palgrave.jors.2600650

Yang, H.L., J.T. Teng and M.S. Chern, 2010. An inventory model under inflation for deteriorating items with stock-dependent consumption rate and partial backlogging shortages. Int. J. Production Econom., 123: 8-19. 